\title{
L'Humanisme en France. État de la question et propositions
}

Jean Céard

\section{(2) OpenEdition}

1 Journals

Édition électronique

URL : http://journals.openedition.org/studifrancesi/9397

DOI : 10.4000/studifrancesi.9397

ISSN : 2421-5856

Éditeur

Rosenberg \& Sellier

Édition imprimée

Date de publication : 1 décembre 2007

Pagination : 516-525

ISSN : 0039-2944

\section{Référence électronique}

Jean Céard, «L'Humanisme en France. État de la question et propositions », Studi Francesi [En ligne],

153 (LI | III) | 2007, mis en ligne le 30 novembre 2015, consulté le 13 janvier 2021. URL : http:// journals.openedition.org/studifrancesi/9397 ; DOI : https://doi.org/10.4000/studifrancesi.9397

\section{(c)}

Studi Francesi è distribuita con Licenza Creative Commons Attribuzione - Non commerciale - Non opere derivate 4.0 Internazionale. 


\section{L'Humanisme en France Etat de la question et propositions}

On ne peut, au moment de présenter un état de la question de l'Humanisme en France, faire l'économie d'une définition du mot. La chose est d'autant plus nécessaire que, parmi les chercheurs qui, en ces trente dernières années, ont le plus contribué à une meilleure connaissance de l'Humanisme, certains, affrontés à l'élasticité de ce concept, ont jugé avoir meilleur compte de s'en passer, comme Marie-Madeleine de La Garanderie, tandis que d'autres en resserraient considérablement l'usage. Si difficile que soit la définition du mot, on ne saurait ici se dispenser de s'y essayer, puisqu'elle engage la délimitation du champ que nous entreprenons de parcourir.

Dans sa Conférence d'ouverture du Colloque de Tours consacré à «L'Humanisme français au début de la Renaissance», il y a quelque trente ans (les Actes en ont été publiés en 1973), V. L. Saulnier observait que l'Humanisme français fait «partie intégrante de l'Humanisme international: profondément philologique, et profane (c'est-à-dire gréco-latin), et, pour le plus, parlant latin»; puis il ajoutait: «Si mêlé, a-t-il le droit au nom d'Humanisme'? [...] L'étude hors théologie, et la préoccupation de modeler l'homme accueillant des nouveaux âges, y tiennent assez de place pour que l'effort puisse se réclamer aussi bien des 'humaniores litterae' que de l' "humanitas". Au total, 'studia humanitatis'»'. On retrouverait aisément ça et là cette définition qui fait apparaître, en même temps qu'elle les lie étroitement, deux sens conjoints du terme d'Humanisme, l'un qui vise la démarche, la méthode, le moyen, et l'autre qui regarde l'objectif, l'ambition, la fin de l'Humanisme. C'est cette étroite subordination de premier sens au second qui fait problème. Quelques brefs rappels historiques permettent de voir l'importance de cette question.

De fait, le terme d'Humanisme a une complexe (quoiqu'assez récente) histoire au cours de laquelle il a tendu à perdre son attache précise avec le temps de la Renaissance pour caractériser toute attitude, toute tendance, toute doctrine à laquelle on veut faire le mérite de mettre l'homme au cœur de ses préoccupations. Mais, par là, il est en quelque sorte victime de ses origines. Faut-il rappeler, ce que tout le monde sait, que le temps de la Renaissance n'a pas connu le mot d'Humanisme, mais seulement celui d'humaniste, terme qui désignait le professeur d'humanités, d'"humaniores litterae", et, en un sens plus large mais dérivé, celui qui se consacrait avec ferveur à l'étude directe des textes antiques? Qu'en ces sens, il soit l'antonyme de théologien, Montaigne, par exemple, en témoigne; quand il écrit son chapitre «Des prières», sujet qui, de tradition, est proprement théologique, et qu'il revendique, lui qui n'est pas théologien de profession, le droit d'en parler, il déclare qu'il a entendu de son temps «faire plainte d'aucuns écrits, de ce qu'ils sont purement humains et philosophiques, sans mélange de Théologie», et il réplique qu'on pourrait non moins justement dire «qu'il se voit plus souvent cette faute, que les Théologiens

(1) L’humanisme français au début de la Renaissance, Paris, Vrin, 1973, p. 25. Voir JEAN CÉARD, V. L. Saulnier, bistorien du premier Humanisme, dans L'bistoire en marge de l'bistoire à la Renaissance, «Cahiers V. L. Saulnier», n 19 (2002), pp. 201-213. 
écrivent trop humainement, que cette autre, que les humanistes écrivent trop peu théologalement $\gg^{2}$. Or, c'est pour avoir un peu trop oublié que Montaigne écrivait en un temps où, selon une longue tradition sanctionnée par l'université, on distinguait les études d'humanité et les études de divinité, qu'on en est venu, dans l'historiographie du XIX ${ }^{e}$ siècle, à interpréter, sans tenir compte de cette opposition de deux termes, bumanior comme un comparatif pour ainsi dire absolu, détournement de sens dont Ferguson crédite justement Hegel. Celui-ci écrivait: «Les hommes se tournèrent vers les ouvrages des anciens [...] comme studia humaniora, où l'homme est reconnu dans ce qui le concerne lui-même et dans ce qu'il effectue»; ou encore: «Le mot humaniora est très expressif, car dans ces œuvres de l'antiquité, honneur est fait à l'humain et au développement de l'humanité»3.

Héritiers de cette historiographie, nous ne pouvons guère nous passer du terme d'Humanisme; mais il importe d'en purifier la notion. À force de vouloir rapporter toute l'activité des humanistes à la fin qu'on leur assignait, on en est venu à ne considérer que comme un modeste préalable leurs études des textes. Certes on ne l'ignore pas - comment pourrait-on faire autrement? Mais, pendant longtemps, on a laissé à quelques laborieux érudits le soin d'examiner la méthode et les résultats de tel ou tel éditeur: dans les synthèses, ce travail n'était guère pris en compte. Qu'on ouvre, à titre d'exemple, l'étude bien connue de M. P. Gilmore intitulée Le monde de l'Humanisme (1952); seul le chapitre VII aborde la question, et encore ne s'agit-il que d'examiner la quête et la découverte de manuscrits et la constitution de bibliothèques; après quoi, l'auteur traite de l'imprimerie: du chemin qui mène du manuscrit à l'imprimé, nulles nouvelles. S'il s'est opéré, disons dans les trente dernières années, un notable changement, c'est bien le fait d'une volonté délibérée de considérer les humanistes au travail, d'analyser leur activité proprement philologique. Cette enquête a conduit à commencer à dresser la bibliographie exhaustive de leurs publications et ainsi à remarquer qu'ils se gardaient de se limiter à l'héritage antique ou même simplement profane, de même qu'ils étaient bien loin de traiter avec le mépris qu'on leur prêtait l'héritage médiéval. Que, de leurs efforts, on puisse dégager une sorte de philosophie de la culture, c'est ce que je m'efforcerai enfin de montrer.

Forcé de nous limiter, nous nous garderons de proposer une bibliographie de ces nouvelles approches. Au reste, l'Humanisme français n'a pas fait l'objet de travaux équivalents, pour l'Italie, à ceux des Sabbadini, des Billanovich ou des Kristeller. On ne saurait pourtant ne pas mentionner une vaste entreprise en cours, dont les premiers travaux ont, du reste, nourri les réflexions ici présentées. Elle est appelée à renouveler profondément notre vision de l'Humanisme. Coordonnée par la section de l'Humanisme de l'Institut de Recherche et d'Histoire des Textes, l'entreprise européenne, intitulée «Europa humanistica», s'est proposé l'étude systématique des conditions de la transmission des textes anciens et médiévaux à la Renaissance. Elle associe douze équipes dans cinq pays européens: Allemagne, Espagne, France, Hongrie, Pays-Bas. D'autres contacts sont actuellement pris, notamment avec l'Italie et la Suisse, en vue de l'extension de ce projet encore jeune, mais qui a déjà donné lieu à des publications ${ }^{4}$.

(2) Montaigne, Les Essais, 1, 56, Paris, «Pochothèque», 2001, pp. 522-523.

(3) W. K. Ferguson, La Renaissance dans la pensée bistorique, trad. fr., Paris, Payot, 1950, p. 162; voir aussi p. 147, n. 2: «Hegel était en partie responsable de la notion erronée d'après quoi bu- manitas ou studia humaniora désignait la littérature dans laquelle l'homme est mis en relief».

(4) En voici la liste:

J. Kecskemeti, M. Portalier, J.-F. Maillard, L'Europe des bumanistes (XIV'-XVII S.) - $2^{\mathrm{e}}$ édition. Turnhout, Brepols, 1998. pp. 544. 
Sans entrer dans les détails techniques de cette vaste entreprise, il est bon de noter qu'elle met l'accent sur la transmission des textes, aspect jusqu'à ce jour trop négligé et qui, en tout cas, n'a pas fait l'objet d'examens d'ensemble. Au reste, elle a opportunément commencé par un volume intitulé L'Europe des Humanistes (1995), répertoire de notices sur 2350 transmetteurs de textes, qui souligne notamment le caractère résolument européen de l'Humanisme, même si chaque Humanisme national a évidemment une coloration propre. Les volumes suivants sont ou seront réalisés par des équipes nationales, selon un protocole commun qui consiste à donner, pour chaque humaniste transmetteur de textes, des éléments de biographie et de bibliographie et la liste des éditions, traductions ou commentaires publiés par un humaniste donné, avec les préfaces et pièces liminaires figurant dans les éditions anciennes. C'est surtout ce dernier point qui fournit de précieuses indications sur les préoccupations, les conceptions, les méthodes, les démarches des transmetteurs de textes.

On les voit certes en quête perpétuelle de manuscrits, ce que nous savions déjà, mais on les découvre surtout conscients de ce qu'il leur appartient de transmettre les textes que les manuscrits ont conservés, travail qui exige, selon des termes qui reviennent sans cesse sous leurs plumes, de les raccoutrer, de les rhabiller, de les rétablir, de les restaurer (instaurare, restituere), c'est-à-dire de les reconstituer. Philippe Montanus, professeur de grec au collège de Tournai, écrit à Charles de Croÿ, évêque de Tournai, qui lui a confié le collège parisien, qu'il en a fait restaurer les bâtiments, et il ajoute que, pour mieux honorer son bienfaiteur, il a entrepris de restaurer, de même, les auteurs anciens ${ }^{5}$. Ces manuscrits sont comme des bâtiments qui menacent ruine, que le temps a détériorés et défigurés, et qu'il s'agit de travailler à remettre dans leur état originel. Il arrive même à ces humanistes de donner la parole aux manuscrits et de prêter une voix aux auteurs, censés attendre anxieusement les restaurateurs qui enfin assureront leur survie. Ce sentiment est fort chez Henri II Estienne: on dirait que, pour lui, ouvrir un manuscrit, prendre connaissance d'un texte, scruter une note, c'est d'abord leur reconnaître leur droit à exister et à perdurer, et en même temps accomplir le devoir sacré d'assurer leur survie. Entrer en contact avec ce manuscrit, ce texte, cette note, c'est exprimer le respect qui leur est dû. Et les publier, c'est inviter ceux qui les liront à s'associer à cette œuvre de respect et de reconnaissance. Tant pis si ces lecteur sont rares, ou s'il faut les attendre des temps à venir. Estienne, qui déplore les misères et les abaissements de son temps, n'est pas loin de penser, comme autrefois Libanios, qu'une nouvelle barbarie est à la porte et qu'il est peut-être le dernier à travailler à sauver ce qui doit l'être.

Comment y parvenir? On peut créditer ces humanistes de la mise au point de ce qu'il faut appeler la philologie critique. Estienne définit ça et là le bon usage des manuscrits ${ }^{6}$. Il sait très bien que tous ne méritent pas la même confiance, mais il observe aussi que tel manuscrit détestable peut suggérer d'excellentes leçons, comme si le copiste avait été comme protégé par sa médiocrité et avait involontairement sauvé

J.-F. Maillakd, J. Kecskemeti, C. Magnien, M. Portalier, La France des bumanistes: Héllénistes, 1, Turnhout, Brepols, 1999 («Europa humanistica», 1).

J. CÉARD, J. KeCSKEMETI, B. Boudou (éds.) H. CAzes, La France des bumanistes: Henri II Estienne, éditeur et écrivain, Turnhout, Brepols, 2003 («Europa humanistica», 2).

W. Kühlmann, Die deutschen Humanisten, Abt. 1, Bd. 1, 1, Marquard Freher, Turnhout, Brepols, 2005.
W. KüHLmann, Die deutschen Humanisten. Abt. 1., Band 1, 2, Janus Gruter, Turnhout, Brepols, 2005.

I. MONOK, E. Zvara, avec la collaboration de E. MÂrZA, Humanistes du bassin des Carpates I, Turnhout, Brepols, sous presse.

(5) Voir La France des Humanistes. Hellénistes I, Turnhout, Brepols, 1999, pp. 330-331.

(6) Je reprends ici quelques remarques de ma Préface à La France des Humanistes. Henri II Estienne, éditeur et écrivain, Turhout, Brepols, 2003. 
telle parfaite relique. Il ne se prive pas de faire des conjectures, dont il est souvent très fier, mais il ne se sent pas autorisé à les introduire dans le texte, comme s'il fallait le garder en l'état en attendant quelque imprévisible circonstance qui permette de lui donner meilleure figure. Il se définit joliment comme le médecin des textes ${ }^{7}$, et il ne serait pas fidèle aux principes de la médecine de son temps s'il ne pensait qu'il ne faut pas bouleverser la manière de vivre du malade et n'intervenir qu'avec une discrète prudence dans l'établissement de son régime. Et, comme le bon médecin suit son malade et l'accompagne, Estienne, qui n'a jamais achevé ce travail de cure, a à peine fini d'imprimer un ouvrage qu'il use des pages blanches qui subsistent pour poursuivre le traitement, ou bien profite d'une autre publication pour y pourvoir. Le cas échéant, une nouvelle édition du même texte permettra de reprendre et de continuer la thérapie. De son côté, Philippe Montanus invente tout un système de signes critiques qui informent le lecteur sur les diverses possibilités de rétablir le texte et le font juge de la qualité de telle ou telle variante. Jean Mercier, en 1556, dans son édition du Promptuaire de droit civil de Harmenopoulos, recourt même à des corps de caractères différents pour distinguer les scolies, les commentaires et les restitutions ${ }^{8}$.

On dit trop souvent que ces humanistes corrigent indiscrètement les textes: ce n'est pas rendre justice à des travaux qui, affrontés à des textes corrompus, cherchent à les améliorer sans nullement dissimuler leurs incertitudes et en mettant explicitement le lecteur en situation de se former sa propre opinion. Le cas de Guillaume Budé annotant les vingt-quatre premiers livres des Pandectes peut donner une idée des méthodes et des considérations qui sont celles des humanistes. Il ne dispose que d'éditions communes du Digeste, de cette sorte de vulgate élaborée par l'École de Bologne et qui, depuis le XII siècle, était en usage dans les universités. Or, presque à chaque instant, on le voit marquer sa défiance à l'égard du texte reçu. C'est l'une des critiques les plus vives qu'il adresse à Accurse, chef de file des glossateurs médiévaux, que d'accepter toujours le texte reçu et même de mettre tout son effort à le conserver: «De quelque texte que se saisisse Accurse, il pense qu'il lui faut le sauvegarder jusqu'au bout, même si les usages langagiers s'y opposent»'. Pour lui, il note que, d'une édition à l'autre, s'observent des variantes parmi lesquelles il se sent alors autorisé à choisir. Si, par exemple, il lit «usus», mais trouve, «dans certains livres», «versus», il retient cette leçon qui lui paraît bien s'accorder au contexte, les anciennes lois, comme celles des Douze Tables, étant écrites en vers ${ }^{10}$. Mais Budé peut aussi, malgré l'accord de toutes les éditions, marquer sa préférence pour une autre leçon: «Ce lieu, écrit-il, dans tous les exemplaires que j'ai vus, se lit peu correctement; pour moi, je pense qu'il faut le lire ainsi», et, il remplace «tum» par «tamen» ${ }^{11}$. Ces corrections, il en fonde la possibilité en se référant aux usages des copistes: il se peut que le texte qu'il lit résulte de la mauvaise résolution d'une abréviation, comme en utilisaient abondamment les juristes ainsi qu'on peut le voir par l'ouvrage de Valérius Probus sur ce sujet ${ }^{12}$. Ailleurs, se référant toujours à ces usages, Budé soupçonne une glose

(7) Ibid., p. 226.

(8) Ibid., p. 286.

(9) Annotationes, Paris, Michel Vascosan, Robert I Estienne et Jean de Roigny, 1542, p. 186: «Accursius vero quemcumque textum arripuit, tuendum ad extremum arbitratur, etiam pugnante loquendi consuetudine».

(10) Annotationes, p. 80: «Sermone etiam antiquo usus affectauit scribere [Dig., I, II, 2, \$46]. Melius in quibusdam libris non usus sed versus legitur. Versus autem intelligendi. XII. tabul».
(11) Annotationes, p. 81: «A Tyberio Caesare hoc tamen illi concessum erat [Dig., I, II, 2, \47]. Hic locus in omnibus quae vidi exemplaribus parum emendate legitur. Ego vero sic legendum arbitror, A Tyberio Caesare hoc tum illi concessum erat».

(12) Annotationes, p. 274: «Aut agrum pollicitatori damus [Dig., XVII, II, 52, \2]. Legendum puto, agrum colendum licitatori damus, id est colono partiario plurimo omnium licitanti. Sic enim fieri solet, ut hanc mendam ex compendio scribendi inoleuisse credam, duabus dictionibus 
d'avoir été introduite dans le texte ${ }^{13}$. Cela dit, même quand Budé estime disposer, grâce à ces corrections, d'un texte satisfaisant, il ne le donne pas pour le texte définitif. Son travail de restitution, il le soumet par principe au contrôle d'une enquête codicologique dont il regrette plusieurs fois de n'avoir pu complètement s'acquitter. Il sait la valeur d'un manuscrit conservé à Pise, puis à Florence, et qui passe pour l'archétype. Étant à Florence, il l'a vu, mais, comme il l'avoue, «à la hâte et, en quelque sorte, à travers un grillage, comme on dit» ${ }^{14}$. Il a eu tout juste le temps de remarquer qu'en bien des endroits il était à demi effacé et que les mots n'y étaient pas interponctués, et de prendre quelques notes, mais il ne doute pas que, s'il avait pu le manier à loisir pendant quelques jours, il y aurait peu de lieux qu'il aurait à laisser incompris, et, sur la foi de cet archétype (tel est le mot par lequel il le désigne couramment), il restitue sans hésitation les passages qu'il a pu examiner. Affronté ailleurs à un passage qu'il ne parvient pas à restituer de façon satisfaisante, il exprime encore son regret de ne pouvoir, sur ce point, consulter «l'archétype de Florence» ${ }^{15}$.

De la philologie critique qu'il met en œuvre, Budé donne ainsi, avec ses Annotationes, une sorte d'exposé par l'exemple. Il présente, non pas tant des résultats, que les cheminements par lesquels il y est parvenu ou, le cas échéant, n'y est pas parvenu. Il veille à donner au lecteur les moyens de juger de la qualité de ses propositions en lui soumettant, dans tout son détail, le dossier qu'il a constitué et en décrivant, dans leur ordre (mêms si c'est sans doute un ordre reconstruit), les étapes par lesquelles il est lui-même passé pour le constituer; et il attend de son lecteur qu'il accueille activement ses propositions: lecteur, Budé veut inciter son lecteur à avoir, à l'égard de celles-ci, l'attitude critique dont son travail donne l'image sensible. Le voici, par exemple, en train d'examiner une loi où il est question d'argentum pusillatum remis à un orfèvre pour fabriquer des récipients. «Qu'adviendrait-il si je lisais pastillatum?», demande Budé ${ }^{16}$. En ce cas, il s'agirait d'argent brut, non affiné, remis à l'orfèvre sous forme des pastilles, la définition de pastille par le lexicographe Publius Festus rendant vraisemblable cette conjecture, qu'on peut fortifier en examinant les emplois pharmaceutiques du mot, mais aussi les usages métallurgiques des anciens que nous

in unam coagmentatis, hoc modo, agrum col licitatori. Fuisse autem morem per notas scribendi in iure ciuili, satis ex Valerio Probo constat. Accursius autem facile deceptus est, propter verbum pollicitus est, quod praecedit».

(13) Annotationes, p. 306: «Castella plumbea putea opercula puteorum epitonea [Dig., XIX, $\mathrm{I}, 17, \mathbb{S} 8$. Lego, castella plumbea putealia, id est opercula puteorum, epistomia. ut fortasse haec verba, id est opercula puteorum, sint ex margine in textum addita».

(14) Annotationes, p. 88: «Nos cum essemus Florentiae, Pandectas Pisanas (quas archetypos esse putant) in palatio vidimus, sed raptim, et quasi transennam, ut dicitur, praetereuntes. Has si aliquot diebus ociose nobis tractare licuisset, pauca, ut existimo, loca non intellecta reliquissemus, tametsi lectu sint perdifficiles, literis iam multis in locis exolescentibus, verbisque non interpunctis».

(15) Annotationes, p. 265, à propos de Dig., XVI, III, 26, \2: «Item costrophinati decem. Sunt qui legant cistophori, quod nummi genus est apud Liuium lib. VII. de bello Maced. [= XXXVII, 46, $3 ; 58,4 ; 59,4]$ quod non probo. nominatiuus enim cistophori, ad verbum debetis, referri non potest. Hic locus igitur vix emendari potest, nisi inspectis archetypis Florentinis. Sunt autem et alia nonnulla loca huiusmodi, quae non tam doctrinae sunt quam fortunae. ut quisque enim aut in antiquissimum aliquod exemplar forte inciderit, aut etiam maiore aliquo fato archetypos legerit, facile haec emendabit, si tamen locus ille incolumis extiterit. sunt enim in iis multa loca in quibus literae vetustate exoluerunt, ut animadvertisse mihi videor cum eas inspicerem».

(16) Annotationes, p. 325: «Veluti cum argentum pusillatum fabro daretur ut vasa fierent $[D i g$. XIX, II, 31]. Quid si pastillatum legam? ut informe argentum infectumque aurifici datum esse intelligamus. Sunt enim pastilli a pane per diminutionem dicti, auctore Festo, massae cuiusque materiae, unde pastilli medicinales. [...] Pastillatum igitur argentum intelligere possumus, in pastillos formatum, cuiusmodi hodie argentum Germani promercale afferunt, quas cendreas appellant. Ac nihilo secius tamen pustulatum legendum contenderim ex Tranquillo in Nerone [XLIV, 2] his verbis, Exegitque ingenti fastidio et acerbitate nummum asperum, argentum pustulatum, aurum obrizum, ut plerique omnem collationem palam recusarent. Ubi pustulatum argentum pro purgatissimo et excoctissimo posuit, cuiusmodi aurum obryzum a Graecis appellatur [...]». 
fait connaître Pline et qui ont cours aujourd'hui encore en Allemagne. Ainsi sont allégués six ou sept textes où l'examen conjoint des mots et des choses semble devoir conduire à retenir la conjecture de pastillatum. Cependant Budé va la rejeter: «Et pourtant, malgré tout, je voudrais soutenir qu'il faut lire pustulatum d'après ce passage de Suétone», passage où l'on trouve l'expression d'argentum pustulatum, et dans un contexte qui permet d'en fixer le sens: il s'agit d'argent purifié au feu, le terme de pustulatum faisant à son tour l'objet d'un examen où est précisée la notion de pustula à l'aide d'une dizaine d'autres textes, et toujours selon la même démarche qui associe l'examen des mots et celui des choses. Notons en passant qu'en ce cas Budé a presque retrouvé la leçon du manuscrit de Florence.

Si je me suis un peu longuement arrêté à l'exemple de Budé, c'est qu'il fait alors figure de maître et que beaucoup d'humanistes se réclament de lui. Car les meilleurs d'entre eux font preuve d'une comparable compétence. Je songe, par exemple, à Josse Bade et à sa très belle édition de l'Institution oratoire de Quintilien, publiée à Paris en 1516. On y observe le même souci de proposer un texte cohérent et de laisser le dernier mot à l'examen codicologique. Bade reconnaît l'extrême mérite de l'édition de Raffaele Regio, dont du reste il reproduit tout le commentaire, chapitre par chapitre, et avant le sien, mais il juge pouvoir parfaire son texte en utilisant un manuscrit qu'il est presque sûr de pouvoir attribuer à Lorenzo Valla, et dont il estime le texte bien supérieur à celui qui a été publié, avec le nom et les commentaires de Valla, en 1494. Donnons un exemple. Quintilien, 1, 10, 22, à propos de la musique, déclare que les nombres que comporte la musique sont également nécessaires à l'orateur: «Num igitur non haec omnia oratori necessaria?» Le texte qu'examine Regius dit: «Num igitur haec omnia oratori necessaria?», sans négation; aussi Regius écrit-il: «Si cette proposition n'est pas corrigée, Quintilien semblera nier ce qu'il entend affirmer. Car une interrogation affirmative appelle couramment une réponse négative. Aussi faut-il y adjoindre l'adverbe de négation non, bien que tous les exemplaria sauf un ancien s'y opposent. 'Num igitur non haec omnia oratori necessaria?' signifie en effet que ce sont choses absolument nécessaires ${ }^{17}$. Bade approuve pleinement cette réflexion, et il est tout heureux de lui apporter la confimation de son manuscrit: «'Num igitur non haec omnia': c'est ainsi que je lis avec Valla; si Regius l'avait vu, il ne l'aurait pas dédaigné» ${ }^{18}$. Cet exemple, en même temps qu'il confirme l'identité des démarches et des méthodes philologiques, montre aussi que ces humanistes ont le sentiment de constituer une communauté et de travailler chacun à son tour à une œuvre commune de restitution.

Là ne s'arrête pas l'exigence de transmission. Je n'ai jusqu'ici cité que des exemples latins. S'il s'agit de textes grecs, les transmettre peut demander qu'ils soient traduits, en latin certes, car le latin est la langue des humanistes, qui considèrent les Latins comme des sortes de compatriotes. Ils ont pourtant pleinement conscience des difficultés de l'entreprise. Ainsi Budé, qui traduit en latin le De placitis philosophorum du pseudo-Plutarque, déclare: «Je sais bien que notre style peut paraître trop dur $(d u$ riorem), mais non peut-être à ceux qui l'ont lu aussi en grec. Il n'est pas facile d'adapter à ce genre de style dense, serré et concis l'éclat et la clarté d'une langue abondante

(17) M. Fabii Quinctiliani oratoriarum institutionum lib. XII, Paris, 1516, f० d $5 \mathrm{r}^{\circ}$ : «Num igitur haec omnia oratori necessaria? Nisi emendetur hic sensus: videbitur Fab. negare quod affirmare contendit. Interrogatio namque affirmativa in negationem solui solet. Quare non negandi aduerbium ascribatur: sicque legatur: quamuis exemplaria omnia praeter vetustum refragentur. Num igitur non haec omnia oratori necessaria? Significat enim maxime necessaria esse».

(18) Ibid.: «Num igitur non haec omnia: sic cum Vallensi lego: quem si vidisset Raphaell non aspernatus esset». 
et coulante» ${ }^{19}$. On sait les querelles des traducteurs de l'époque, notamment celle que suscitèrent les traductions latines d'Aristote de Joachim Périon. Il n'échappe pas à ces traducteurs que la traduction n'est pas seulement une affaire de style, que l'on court le risque majeur de trahir la «sententia viaque» de l'auteur, comme l'écrit Jacques Louis d'Estrebay, en 1542, en tête de sa traduction de la Politique d'Aristote ${ }^{20}$.

Laissons ces problèmes de la traduction, qui demanderaient un beaucoup plus long examen, pour aborder l'étape suivante de la transmission, qui est la publication. On a eu raison de souligner que le travail des humanistes est antérieur à l'invention de l'imprimerie, mais ajoutons tout de suite qu'ils ont salué cette invention comme une grâce divine, par laquelle la diffusion du savoir qu'ils élaboraient se trouvait infiniment facilitée. S'il est des humanistes pour rappeler, avec une amertume d'intellectuels, que c'est le souci du gain, non l'érudition, qui meut certains imprimeurslibraires, il faut aussi noter que beaucoup de ceux-ci sont d'authentiques savants; le cas le plus évident (mais ce n'est pas le seul) est celui d'Henri Estienne, savant et imprimeur-libraire: on sait les difficultés matérielles où l'engagea son édition du Thesaurus de la langue grecque. Les préfaces et pièces liminaires, en même temps qu'elles fournissent une foule d'informations sur le monde du livre, font aussi apparaître le souci permanent des humanistes d'atteindre un plus grand nombre de lecteurs. Ainsi l'érudit Estienne ne travaille pas pour les seuls érudits. Il a publié et lui-même composé des «colloques» scolaires et il invente un format d'édition à l'intention de lecteurs débutants. S'il a certes besoin, comme on dit aujourd'hui, de rentabiliser son entreprise, on ne saurait manquer d'ajouter que de telles publications ressortissent, elles aussi, à une volonté d'atteindre un large public et de ne pas laisser les richesses de l'antiquité aux seules mains d'un tout petit nombre. Se rappelant combien le grec l'a lui-même éveillé et formé, Estienne croit profondément à leur pouvoir d'éveil et de formation. A lui d'en procurer les moyens.

Que ces humanistes soient persuadés que l'héritage antique a ce pouvoir, on ne saurait le nier. Mais encore faut-il nous montrer attentifs à ce qui, pour eux, constitue cet héritage. Que l'on considère la production imprimée du temps de la Renaissance, et l'on constatera la présence massive de la patrologie, au sens le plus large de ce terme. Budé, s'il transmet Aristote, Démosthène, Plutarque, Végèce et Xénophon, transmet aussi Philon et Basile de Césarée. Estienne transmet aussi Anastase, Athanase, Athénagoras, Basile de Césarée, Cyrille d'Alexandrie, Grégoire de Nazianze, Justin, et d'autres. Montanus transmet Jean Chrysostome et Théophylacte. On multiplierait aisément les exemples. Cet intérêt s'étend, on le sait aussi, à l'hébreu: Agostino Giustiniani (qui, lecteur royal à partir de 1518, appartient au moins en partie à l'Humanisme français) publie Maïmonide et Moïse Kimhi; rappelons-nous encore les travaux de Jean Mercier, de Chéradame et de beaucoup d'autres. Enfin comment négliger la place massive des études bibliques dans les travaux des humanistes? Tous ces faits sont bien connus: c'est leur appréciation qui fait problème. Nous avons tellement l'habitude d'opposer les humanistes et les théologiens qu'ayant pour ainsi dire hypostasié l'idée d'humaniste, et la justifiant par la considération des 'litterae humaniores', il nous semble que l'Humanisme est fondamentalement profane et que les humanistes devraient se limiter à «l'étude hors théologie»; si donc on les voit se préoccuper de la Bible et des Pères, c'est sans doute, pose-t-on, par pure préoccupation philologique.

Que le souci philologique ne les quitte pas, Budé en témoigne aisément. Déjà les Annotationes (1508) montrent une nette attention aux textes sacrés dont Budé estime 
qu'ils doivent être établis et analysés selon les mêmes règles que celles qu'il applique aux textes de droit romain. De même qu'il critique Accurse qui veut à tout prix sauvegarder jusqu'au bout le texte reçu, de même il blâme ces lecteurs de l'Écriture qui tiennent la Vulgate pour sacro-sainte ${ }^{21}$, qui pensent que cette traduction, parce qu'elle est depuis longtemps reçue par toute l'Eglise d'Occident, dispose d'une autorité devant laquelle doit s'effacer le texte original ${ }^{22}$. Et il s'efforce de le prouver en examinant divers passages du Nouveau Testament et des parties originellement grecques de l'Ancien Testament. Le dernier grand livre de Budé, le De transitu Hellenismi ad Christianismum (Le passage de l'bellénisme au christianisme), publié en 1535, s'ouvrira encore par l'idée de philologie. Il y distinguera la philologia minor, qui s'applique aux «studia humanitatis», c'est-à-dire à la culture profane, et la philologia maior, dont l'objet est l'interprétation et la méditation des textes sacrés, et le dessein, la recherche de ce qu'il appelle la sagesse, laquelle est toute contenue dans l'Écriture Sainte. Sans étudier ici les développements que Budé donne à ce projet, on se contentera ici de souligner qu'il n'implique nullement un renoncement aux «études d'humanité», puisque Budé indique qu'il a résolu de «transférer» à l'étude de la sagesse «toutes les ressources et les capacités» dont il dispose et que les études d'humanité lui ont permis d'acquérir et d'exercer. Il n'entend aucunement, dit-il, «renoncer à l'usage de cette ancienne philologie que j'ai acquise et pratiquée si longtemps ${ }^{23}$. S'interrogeant, dès le De asse (1515), sur la question cruciale de la relation des lettres profanes et des «saintes lettres», Budé médite sur l'exemple de Salomon (Ecclésiaste, 16, 26). Comme Salomon, dit-il, il faut commencer par faire le tour de tout («Lustraui uniuersa animo meo», dit la Vulgate): «Ceux qui de nos jours se disposent à étudier la philosophie feront plus sagement de ne pas s'adonner à elle immédiatement au sortir des rudiments, mais, tels des chasseurs actifs et énergiques, de commencer par quêter les traces de la sagesse à travers les monuments de toute espèce de science [...]. Les oiseaux qui s'envolent vers un sommet abrupt ne le gagnent pas en ligne droite, mais s'élèvent bien plus aisément en tournoyant. [...] C'est en ce sens que Salomon [...] semble nous exhorter à recueillir à travers tous les systèmes de la philosophie profane et ancienne les traces de la sagesse» ${ }^{24}$. C'est en somme la pensée des Pères et surtout de S. Augustin (De doctrina christiana, XL), mais exprimée à l'aide de parlantes métaphores qui dessinent un mouvement ascensionnel, nécessaire, des lettres profanes à la sagesse de Dieu. C'est aussi celle de Basile de Césarée dans sa Lettre aux jeunes gens, et il n'est pas surprenant que l'œuvre de ce Père ait retenu l'attention de nombreux éditeurs. Que tous les humanistes français n'aient pas aussi fortement que Budé cette exigence

(21) Annotationes, éd. citée, p. 74: «Nec vero ignoro homines undique reclamitaturos, utpote opinione praesumpta auctoritatis tantae interpretis (quem ipsi falso Hieronymum fuisse credunt) receptaeque iam tot seculorum consensu huius nostrae interpretationis, quam ut sacrosanctam attingi non posse sine ingenti piaculo multi vociferantur. Quod vero ad me attinet, sancrosanctam prorsus Lucae historiam puto, qui ipse graece scripsit».

(22) Ibid., p. 315: «Ridebunt illi qui evangelia Graeca, et totum instrumentum nouum fidei nostrae, id est Apostolorum et Euangelistarum editionem, nullius auctoritatis esse credunt, praeiudicio illo utentes, quod haec nostra traductio ab uniuersa ecclesia occidentis recepta sit. Quasi vero errata etiam nominatim recepta sint, aut quasi haec traductio publico decreto Graecae origini praelata sit, $a b$ iis praesertim qui Graece prorsus nihil sciue- runt, id est qui post Hieronymum, Ambrosium et Augustinum fuerunt. Quod simile est atque si traduces ex vite aut arbore ductas, praestantiores stirpibus ipsis esse contendant unde sint enatae».

(23) G. BudÉ, De transitu bellenismi ad christianismum, éd. et trad. M.-M. de LA GARANDERIE et D.F. Penham, Paris, Les Belles Lettres, 1993, Préface, p. 1: «Ad hoc ipse alterum studium [...] transferre eundem animum concupiui, copias item ipsius omnes, facultatesque transcribere, quales illae sunt cunque. Id demum receptum volui, cautumque diligenter, ne mihi necesse esset usum et consuetudinem philologiae pristinae, contractam perdiu et conformatam, sic ipsi renunciare, eam ut ipsam ultimum valere iuberem, semelque salutarem».

(24) G. Budé, De Asse, Paris, Vascosan, 1541, f. CCIIb. 
d'une sorte de progrès spirituel qui fait des lettres profanes une sorte de propédeutique de la sagesse, c'est probable; mais il est permis de demander aux plus grands d'entre eux de la formuler. Au reste, diverses publications d'humanistes font bien voir qu'au moins ils demandent aux païens de contribuer à servir la vérité chrétienne telle qu'ils l'entendent: quand Gentian Hervet procure, en 1544, une édition du De fato d'Alexandre d'Aphrodise, il souligne l'utilité de telles œuvres pour combattre la doctrine hérétique de la prédestination ${ }^{25}$.

Que l'on considère la voie immédiatement polémique d'Hervet ou celle, infiniment plus large et patiente, de Budé, dans les deux cas leurs positions postulent une certaine unité de la culture. Une unité qui apparaît à qui, tout en respectant et en pratiquant même les recherches les plus spécialisées, garde en ligne de mire la totalité du savoir dans la diversité de ses réalisations comme dans l'histoire de sa constitution. Puisque cette rencontre de Turin est dédiée à la mémoire de Franco Simone, comment ne pas rappeler ici l'étude fondatrice qu'il a consacrée à «la notion d'encyclopédie: élément caractéristique de la Renaissance française ${ }^{26}$ ? Il avait notamment cité le texte de Budé qui vient d'être rappelé, et où celui-ci ajoutait que Salomon «avait parcouru du mouvement circulaire de l'encyclopédie (encyclopediae gyro) tous les monuments des grands esprits». Le mot a été très précisément défini (et curieusement traduit) par Budé lui-même dans son Institution du prince, où il parle d'«une perfection des arts liberaux et sciences politiques qu'on appelle en Grec Encyclopedia, qui vaut autant à dire (pour le declairer briefvement) comme erudition circulaire, ayans lesdictes sciences et disciplines connexité mutuelle et coherence de doctrine et affinité d'estude, qui ne se doibt ny peult bonnement separer ny destruire par distinction de faculté ou profession [...], pource que toutes les sciences s'entretiennent comme font les parties d'un cercle qui n'a ny commencement ny fin, et toutes tendent de leur naturelle inclination vers le centre du cercle, lequel centre nous pouvons icy imaginer estre congnoissance du bien souverain et desir de parvenir à icelluy» ${ }^{27}$. C'est une idée sans cesse présente à l'esprit de Budé. Sans prononcer alors le mot, il note, dans ses Annotationes, que, pour comprendre vraiment le droit, il faut savoir le reste, car la science du droit est une partie de la science civile, qui est elle-même une partie de la science morale, laquelle est une part de la philosophie ${ }^{28}$. Voilà le centre vers lequel tend le droit.

Ainsi conçue, l'encyclopédie n'est pas tant la clôture circulaire du savoir que l'effort par lequel le savoir s'organise en cercle, tâche à laquelle on ne saurait assigner un terme. L'encyclopédie ne peut cesser d'être projet. Elle se dénaturerait en prenant la forme d'un exposé qui, classant, ordonnant et subordonnant, offrirait, quelle qu'en soit la qualité, un panorama arrêté du savoir. Pour que le savoir reste ordonné à sa fin, qui est la sagesse, il doit garder l'élan qui lui a donné le branle, demeurer animé par l'esprit d'enquête qui le conduit. Composer une encyclopédie, quel que soit le mode de son ordinatio, ce serait arrêter l'élan, exténuer l'esprit d'enquête. Complémentairement, s'attarder trop, note Budé, dans la forêt de l'encyclopédie (encyclopediae sylua) reviendrait à s'obstiner à y ramasser les quelques glands qui y restent, au moment où il importe de conduire ses pas dans ce champ de blé qu'est le champ du Seigneur ${ }^{29}$.

(25) La France des Humanistes. Hellénistes I, cit., p. 194.

(26) Dans French Renaissance Studies (15401570). Humanism and the Encyclopedia, éd. P. SHARRATT, Edinburgh, U.P., 1976. p. 88 .
(28) Annotationes, éd. citée, p. 281: «Iuris scientia, ut alibi diximus, pars est ciuilis scientiae, quae rursus ipsa pars est moralis scientiae. Porro moralis scientia, tertia pars est philosophiae, etiam cum philosophia angustissimis finibus circunscribitur».

(29) Budé, De transitu [...], cit., p. 252. 
Néanmoins, on le voit bien par ces riches réflexions de Budé, il n'est à aucun moment question de récuser les lettres profanes, les 'litterae humaniores'. Nul regret chez Budé de leur avoir accordé une si longue et si intense attention. Car les lettres profanes enregistrent les cheminements des hommes vers la sagesse, qui les couronne et les dépasse; et la philologia minor prépare à son tour les esprits à accéder à la philologia maior. Si être humaniste, c'est affirmer la nécessité de la philologia minor en vue de cette fin, et donc la dignité des lettres profanes, Budé est pleinement un humaniste; c'est en ce sens, purifié, que le terme d'Humanisme mérite d'être conservé.

Forcé de me limiter, je n'ai à peu près pas touché à un ensemble de sujets connexes: les informations que fournissent préfaces et pièces liminaires sont aussi extrêmement riches sur le monde des humanistes, avec leurs protecteurs, leurs mécènes, leurs collègues, leurs amis et leurs adversaires, sur leur intérêt pour la chose publique, pour les institutions culturelles et pour l'enseignement, par exemple. Mais les premiers sujets regardent la vie intellectuelle en France au XVI ${ }^{\mathrm{e}}$ siècle, et ne sont pas spécifiquement humanistes. Les seconds, qui regardent davantage l'Humanisme, sont au fond des applications de la philosophie de la culture dont j'ai dégagé quelques traits. Il resterait à en examiner les modes d'expression. J'ai mis l'accent sur le travail de transmission des textes. Il faudrait aussi étudier les formes que prend l'appropriation des textes, et surtout la principale d'entre elles, le commentaire, qui, avec l'Humanisme, se diversifie et se transforme profondément: outre le commentaire critique que constitue l'établissement des textes, le commentaire peut prendre la forme d'annotationes, de lectiones, ou encore de dictionnaires, qui sont bien encore des commentaires: on peut citer les Commentarii linguae graecae de Budé ou les Commentaria linguae latinae d'Estienne Dolet. En outre, s'il est vrai que le savoir antique a valeur propédeutique, il conviendrait d'examiner les façons dont les textes sont interrogés, pour y percevoir ces «traces de la sagesse», ces «vestigia sapientiae», dont parle Budé, notamment dans les mythes antiques. Ronsard, s'il n'est pas transmetteur de textes, est bien humaniste dans la façon dont, sous l'impulsion de Dorat, il interroge les mythes. Le mythe de Mercure chez Budé, les mythes d'Hercule ou de Bacchus chez Ronsard, le mythe très répandu de l'Hercule Gaulois sont aussi des témoins de cette philosophie de la culture qui anime et définit l'Humanisme.

Que l'Humanisme ait nourri et vivifié la pensée de la Renaissance, en France comme hors de France, ne saurait être contesté. Il est bien évident que de grands thèmes, comme celui de la dignité de l'homme, si minutieusement étudié par Lionello Sozzi ${ }^{30}$, ont été, sinon portés au jour, du moins considérablement alimentés et, pour ainsi dire, orientés par les travaux des humanistes. Mais, à moins d'appeler Humanisme la totalité de la pensée de la Renaissance, c'est-à-dire de diluer l'Humanisme et de lui retirer, de fait, toute sa force innovante, il est profitable, et historiquement pertinent, de le considérer comme un moment de la vie de l'esprit qui, en rendant aux lettres profanes leur pouvoir séminal et en élaborant une philosophie originale de la culture, fondée sur la riche et féconde idée de la philologie et sur celle de l'unité profonde du savoir, a changé les conditions de l'exercice de la pensée. C'est à lui que l'on doit ce que Gilbert Gadoffre a justement appelé la «révolution culturelle» de la Renaissance ${ }^{31}$.

JEAN CÉARD

(30) Voir notamment L. Sozzi, Un désir ardent. Etudes sur la Dignité de l'bomme à la Renaissance, Torino, Il Segnalibro, 1998.
(31) G. GAdOFFre, La révolution culturelle dans la France des bumanistes. Préface de Jean CÉARD, Genève, Droz,1997 («Titre courant»). 